\title{
Active-Region Sources of Solar Wind near Solar Maximum
}

\author{
Paulett C. Liewer*, Marcia Neugebauer*\# and Thomas Zurbuchen $\dagger$ \\ *Jet Propulsion Laboratory, California Institute of Technology, Pasadena, CA 91109 \\ *\# Lunar and Planetary Laboratory, University of Arizona, Tucson, AZ 85721 \\ †University of Michigan, Space Physics Research Laboratory Ann Arbor, MI 48105
}

\begin{abstract}
Previous studies of the source regions of solar wind sampled by ACE and Ulysses showed that some solar wind originates from open flux areas in active regions. These sources were labeled active region sources when there was no corresponding coronal hole in the He $10830 \AA$ synoptic maps. Here, we present results on an investigation of the magnetic topology of these active region sources and a search for corresponding features in EUV and soft X-ray images. In most, but not all, cases, a dark hole or lane is seen in the EUV and SXT image as for familiar coronal hole sources. However, in one case, the soft-X ray images and the magnetic model showed a coronal structure quite different from typical coronal hole structure. Using ACE data, we also find that the solar wind from these active region sources generally has a higher Oxygen charge state than wind from the Helium-10830 $\AA$ coronal hole sources, indicating a hotter source region, consistent with the active region source interpretation.
\end{abstract}

\section{INTRODUCTION}

The sources of solar wind at solar minimum are understood to some extent: fast wind ( $\mathrm{V}>700 \mathrm{~km} / \mathrm{s})$ comes from the large polar coronal holes and slow wind $(\mathrm{V}<600 \mathrm{~km} / \mathrm{s})$ comes from a broad region surrounding the heliospheric current sheet and also from equatorial coronal holes (Neugebauer et. al., 1998).

Recently, Neugebauer et. al. (2002, hereafter Paper I) reported results of a study of the sources and properties of solar wind near solar maximum. The photospheric sources were determined using a magnetic mapping procedure. We found that much wind sampled by ACE and Ulysses near solar maximum originated from open field regions in or adjacent to active regions. When the calculated source was a region of high field strength and there was no corresponding coronal hole in He $10830 \AA$ synoptic map, these sources were labeled active region sources to distinguish them from the traditional "coronal hole" sources that show a coronal hole in NSO He $10830 \AA$ synoptic maps. The source region temperature of these active region sources, as determined from the solar wind Oxygen charge state data, was found to be higher than for coronal hole source regions, consistent with an active region source identification. No other property investigated, including velocity, showed a statistically significant difference between coronal hole and active region sources.
While others have concluded that some open flux and solar wind originates in active regions (Levine, 1977; Kojima et. al, 1999), Paper I was the first to use in situ data to show that wind from open flux in active regions has distinctive properties (e. g., more variability, higher freezing-in temperature).

Here we report a more detailed study of several of the active regions sources that were part of the statistical study in Paper I. Specifically, we ask if these active regions sources are evident as dark features in EUV and soft X-ray images (see Levine, 1977). We study the topology of the corona above these active region sources using both the images and potential magnetic field models. We also compare the Oxygen charge state of wind from active region and coronal hole sources and find distinct signatures. Using these signatures, we find that we are sometimes able to determine the type of source regions in cases where the magnetic mapping alone was ambiguous

It is known that $\mathrm{He} 10830 \AA$ is an imperfect diagnostic for determining the footpoints of coronal open flux. Because of this, some would argue that open flux sources of solar wind in active regions should also be called coronal holes. However, because the wind from these active-region sources shows different properties than wind from traditional "coronal holes," we use the term active region source for these open flux sources in active regions with no corresponding hole in He $10830 \AA$ synoptic maps. 


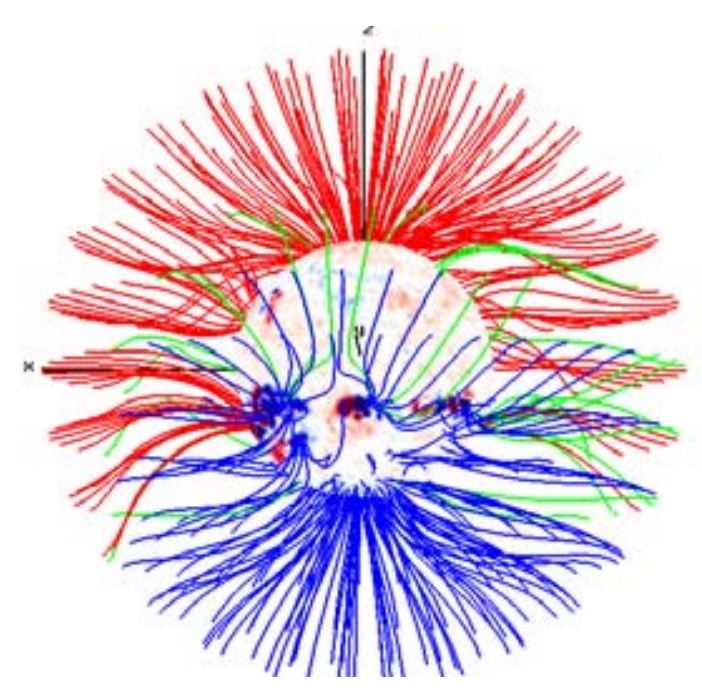

FIGURE 1. CR 1934 magnetogram and magnetic model showing the mapping of open flux from the source surface $\left(\mathrm{R}=2.5 \mathrm{R}_{\mathrm{S}}\right)$ to the photosphere. Red (blue) is inward (outward) polarity; green lines lie near the current sheet. April 7, 1998.

\section{DETERMINING SOURCE REGIONS}

The photospheric sources of solar wind sampled by ACE is determined using a two-step mapping process as in Neugebauer et al. (1998). First, the solar wind speed measured at ACE is used to ballistically map the solar wind back to a source surface, $R=2.5 R_{s}$ (solar radii), assuming the wind travels radially at the measured velocity from the source surface to ACE.
In the second step, a potential field source surface model extrapolated from an NSO Kitt Peak synoptic magnetogram is used to trace the solar wind from the source surface to the photosphere, assuming the wind follows the magnetic field lines. Further details can be found in Paper I. We check each mapping against three criteria: (1) Does the potential field model predict open flux where the NSO He $10830 \AA$ synoptic maps show coronal holes? (2) Does the polarity of the magnetic field at the spacecraft agree with the polarity predicted by the mapping? (3) Does the solar wind data show evidence of a source region boundary at the locations predicted by the model? Paper I analyzed the mappings for four Carrington rotations where these criteria were generally well satisfied. Here, we further investigate the active region sources from these rotations. While the potential field models show the open flux of the He $10830 \AA$ synoptic coronal holes, the models often show additional small regions of open flux in active regions with no corresponding $\mathrm{He}$ $10830 \AA$ coronal hole in the synoptic maps.

\section{Mapping Results for CR1934}

Figure 1 shows open flux field lines for the potential field source surface model computed for Carrington rotation 1934 (corresponding to days 77104 of 1998 at the Sun). The field lines were generated by tracing field lines from the source surface to the photosphere; the lines are uniformly spaced in area at the source surface. Note that open flux comes from both the polar coronal holes and the active region belt.

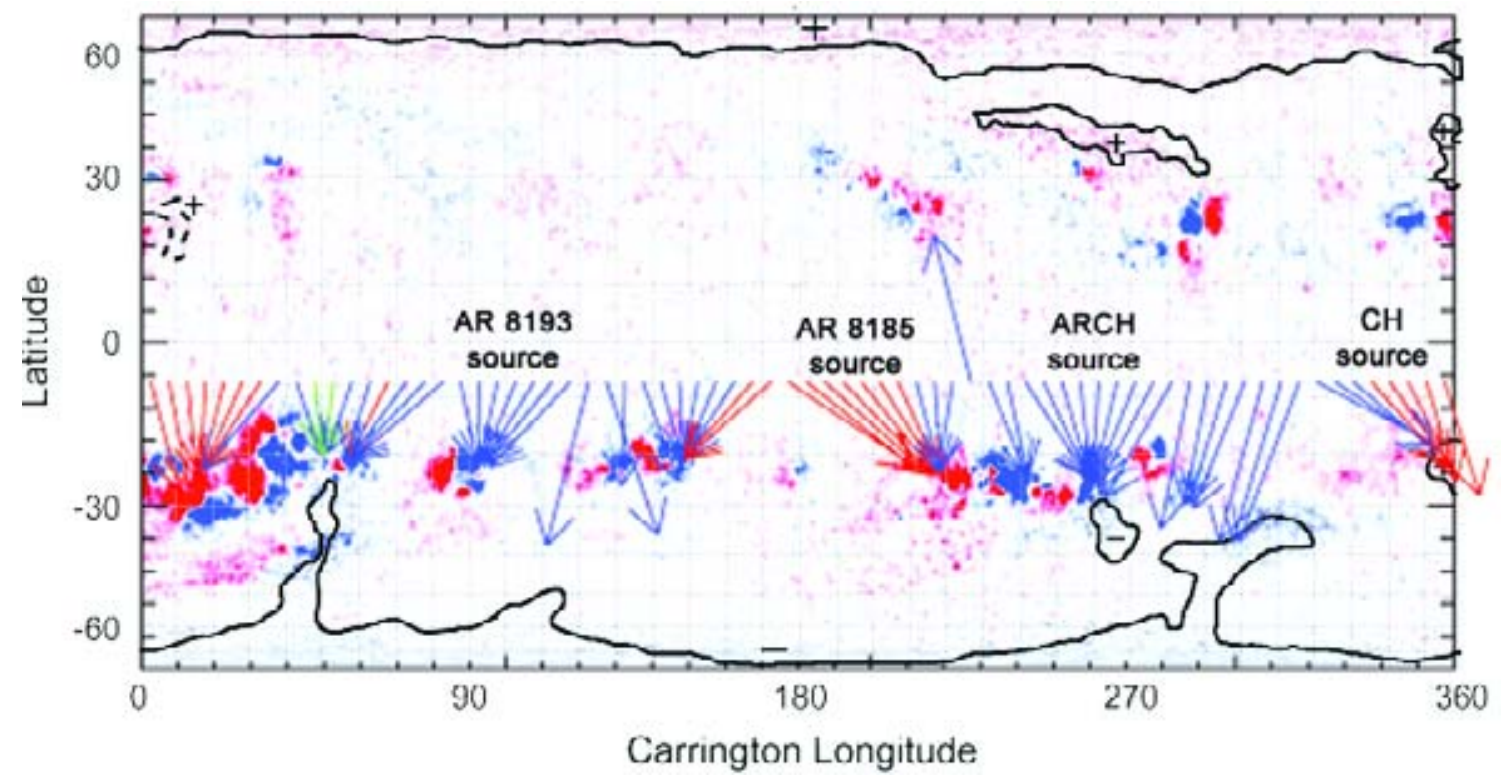

FIGURE 2. Carrington rotation 1934 magnetogram, synoptic coronal holes and arrows mapping ACE solar wind from the source surface (arrow tails) to the photosphere (heads). Several different sources and streams are evident. The two active-region sources $(\mathrm{AR})$, one coronal hole source $(\mathrm{CH})$, and one indeterminate $(\mathrm{ARCH})$ source are discussed in the text. 

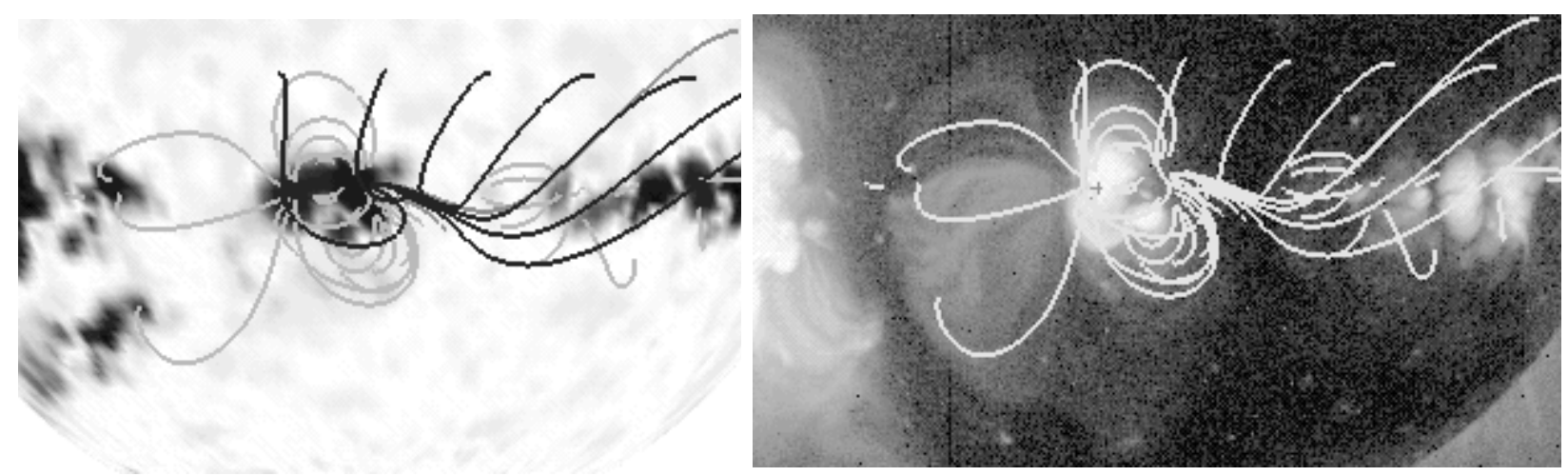

FIGURE 3. Left: Magnetic model of active region AR 8193. The dark lines are the open field lines of ACE solar wind that mapped to AR 8193 (see Fig. 2). The open flux comes from one outer edge of the bipolar AR 8193. The light lines are closed field lines. View as in Fig. 1: April 7, 1998. RIGHT: Yohkoh SXT image with the model magnetic field lines of the left panel superimposed. The open flux is at the edge of the dark lane seen by SXT (April 7, 1998 12:51 UT).

In Figure 2, the arrows showing the potential field mapping of the ACE solar wind from the source surface $\left(\mathrm{R}=2.5 \mathrm{R}_{\mathrm{S}}\right)$ to the photosphere overlie the synoptic magnetogram. The arrow tail is the solar wind field line location at the source surface (as determined from the ballistic mapping) and the head is at the photospheric source (field line footpoint). Also shown are the coronal holes from the He $10830 \AA$ synoptic maps. The field polarity measured at ACE agrees well with that predicted by the model. Note that most of the sampled wind comes from the active region belt even though the polar coronal holes are still present. Several different sources and streams are evident from the bunching of the arrow heads.

In this rotation, we identify two active regions sources (see Fig. 2). The stream with source surface location between $90^{\circ}-110^{\circ}$ longitude is on open field lines that trace to active region AR 8193. The wind with source surface longitude $180^{\circ}-220^{\circ}$ is on open field lines that map to active region AR 8185. Neither source region has a synoptic $10830 \AA$ coronal hole nearby. On the other hand, the wind from $250^{\circ}-280^{\circ}$ longitude maps to a strong field region with an adjacent synoptic $10830 \AA$ coronal hole. Because the mapping is not accurate enough to discriminate between open flux from the active region or from the adjacent coronal hole, we label this an ARCH source to indicate the uncertainty. Wind from $320^{\circ}-360^{\circ}$ is on open flux from the synoptic $10830 \AA$ coronal hole at $\sim 360^{\circ}$ and this is labeled a $\mathrm{CH}$ source region. Below, we look at topology of the two AR source and solar wind from all four sources.

\section{Topology of the Active Region Sources}

The left panel of Figure 3 shows the magnetic topology of AR 8193 as computed from the potential field model; the view corresponds to the Earth's view of the Sun on April 7, 1998. The dark lines are the open field lines of ACE solar wind that mapped to AR 8193 (see Fig. 2). The light lines are closed field lines. Note that the open flux comes from an outer edge of the bipolar active region, e.g. the edge away from the opposite polarity region of the same active region. The right panel of Figure 3 shows these same field lines superimposed on a Yohkoh SXT image from April 7, 1998. Note that the open flux lines from the active region are at the edge of a dark lane in the SXT image. Dark lanes in Skylab images were also associated with open flux by Levine (1977). Here, the dark lane is much larger than the photospheric region with open flux. For this AR source, there was no coronal hole in either the daily or the synoptic $10830 \AA$ maps.

The magnetic topology of the other active region source for this rotation, AR 8185, was similar in that the open flux came from the outer edge of the active region. In this case, a transient coronal hole appears in daily $10830 \AA$ maps for March 24-27. The transient coronal hole is also evident as a dark hole in both the SOHO EIT and Yohkoh SXT images for March 28, 1998 when AR 8185 was near central meridian

We have also looked in detail at two other AR sources from other rotations analyzed in Paper I. AR 8681 in CR 1953 also shows open flux coming from an outer edge of the active region. A very small transient coronal hole is seen in the daily $10830 \AA$ maps. A region much larger than this transient coronal hole is seen as a dark lane in the EIT and SXT images for August 29, 1999 when the active region was near the central meridian. For the above three active region sources, the open flux comes from an outer edge of the active region and a dark hole or lane in the SXT and EIT images can be associated with the open flux.

In contrast, the active region in CR 1953 has a different topology (not shown). Here, two neighboring bipolar strong field regions form a dual dipole; the 
open flux comes from the interior of one polarity region where both the magnetic model and the Yohkoh SXT image show a magnetic separatrix: To one side of the open flux, the loops connect eastward (to the opposite polarity region of the same bipolar regions), and on the other side of the open flux, the loops connect to the west (to the opposite polarity region of the neighboring bipolar region). The separatrix can be well seen in the SXT images of December 18, 1999 as a somewhat dimmer "part" in the loop structure, but no dark feature was evident. Thus, not surprisingly, there appear to be a variety of magnetic topologies that allow open flux from active regions.

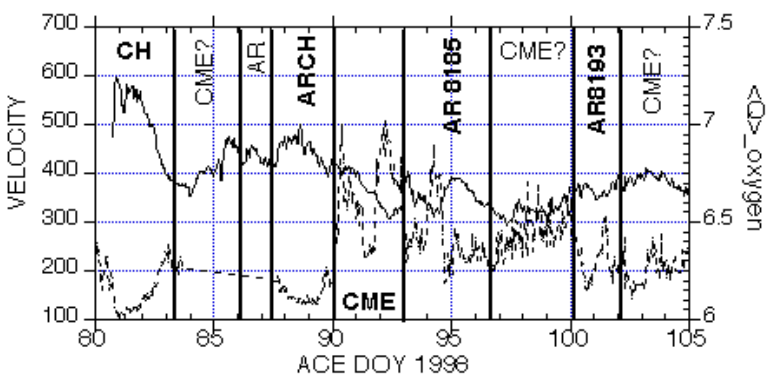

FIGURE 4. ACE solar wind proton velocity (solid) and average Oxygen charge state $<Q>$ (dashed). The vertical lines separate wind from different sources, determined from the mapping (Figure 2). Carrington longitude goes from right to left: Day 80 maps to $\sim 360^{\circ}$ and Day 105 maps to $55^{\circ}$.

\section{Solar Wind Charge State Data}

Figure 4 shows ACE solar wind data [proton velocity (solid line) and average Oxygen charge state $<$ Q $>$ (dashed line)] for ACE days 80-105 of 1998, covering the four source regions labeled in Figure 2 (as well as the intervening streams). Day 80 maps to $\sim 360^{\circ}$ Carrington longitude and Day 105 maps to $55^{\circ}$. These data are typical of the four rotations studied. Note the low Oxygen charge state in the wind from the coronal hole (ACE days 80-83); here, the minimum $<\mathrm{Q}>$ is close to 6 , similar to wind from polar coronal holes at solar minimum. The speed of the wind from this coronal hole, however, is somewhat low, $\mathrm{V}<600$ $\mathrm{km} / \mathrm{sec}$. Note that $<\mathrm{Q}>$ is significantly higher and more variable in the wind from the two active regions, AR 8185 and AR 8193. The wind from these active region sources also appears to be composed of multiple substreams as noted in Paper I. Both characteristics are consistent with our active region source identification. The wind from active regions may have been heated on closed field lines which opened by reconnection, allowing the plasma to escape. Such an interpretation is consistent with that of Zurbuchen et al. (2000) who concluded that slow wind with higher charge states emerged from closed coronal magnetic structures.
Note in Figure 4 that the value and behavior of $<Q>$ in the ARCH wind is clearly similar to that of coronal hole wind. Using this information, we can now assume that this wind is from the small coronal hole at $260-270^{\circ}$ longitude and not the nearby active region. Thus the charge state data allows us to remove some ambiguities in the mapping procedure.

\section{CONCLUSIONS}

We have studied active region sources of solar wind. Our study of the topology shows that in most cases, a dark hole or lane is seen in the EUV and SXT image at the location of the active region source, as for familiar coronal hole sources. In one case, when the magnetic model showed the open flux was associated with a separatrix within a strong field region, the soft$\mathrm{X}$ ray images did not show a dark lane or hole. Here, the separatrix was evident in the X-ray loop structure. Using ACE data, we find that the solar wind from these active region sources generally has a higher Oxygen charge state than the coronal hole wind, indicating a hotter source region, consistent with the active region source interpretation.

\section{ACKNOWLEDGMENTS}

We thank Z. Mikic, SAIC, for the potential magnetic field and field line tracing programs used in this work. Much of this work is the result of research performed at the Jet Propulsion Laboratory of the California Institute of Technology under a contract with NASA. The University of Michigan work was also supported by NASA.

\section{REFERENCES}

1. Neugebauer, M., et al., Spatial structure of the solar wind and comparison J. Geophys. Res., 103, 14587 (1998).

2. Neugebauer, M., Liewer, P. C., Smith, E. J., Skoug, R. M., and Zurbuchen, T. H., Sources of the Solar Wind at Solar Activity Maximum, J. Geophys. Res. (2002).

3. Levine, R. H., Large scale solar magnetic fields and coronal holes, in Coronal Holes and High Speed Wind Streams, ed. J. B. Zirker, pp. 103-143, Co. Assoc. Univ. Press, Boulder (1977).

4. Kojima, M., K. Fujiki, T. Ohmi, M. Tokamaru, A. Yokobe, and K. Hakamada, Low-speed solar wind from the vicinity of solar active regions, J. Geophys. Res., 104, 16993 (1999).

5. Zurbuchen, T. H., S. Hefti, L. A. Fisk, G. Gloeckler, and N. A. Schwadron, Magnetic structure of the slow solar wind: Constraints from composition data, J. Geophys. Res., 105, 18327 (2000). 